\title{
Use of Adjuvant Endocrine Therapy Among Post-Menopausal Breast Cancer Patients in Malaysia
}

\author{
Athirah Saiful Bahri \\ Tuan Mazlelaa Tuan Mahmood \\ Siti Azdiah Abdul-Aziz \\ Mohd Makmor-Bakry (D) \\ Noraida Mohamed Shah (DD \\ Centre of Quality Management of \\ Medicines, Faculty of Pharmacy, Universiti \\ Kebangsaan Malaysia, Kuala Lumpur, \\ Malaysia
}

This article was published in the following Dove Press journal: Patient Preference and Adherence

Context: Globally, breast cancer is the most common cancer affecting women, and adjuvant endocrine therapy (AET) is part of its treatment modality, which improves patients' outcome. However, there are concerns on side effects related to its use, which may affect treatment adherence.

Purpose: This study was conducted to explore attitude and practice on using AET among breast cancer patients in Malaysia.

Patients and Methods: Postmenopausal breast cancer patients on at least 3 months of AET attending the outpatient oncology clinic at a tertiary care hospital were interviewed. Patients underwent in-depth interviews exploring their attitude and practices while on AET using a semi-structured interview guide. The interviews were transcribed verbatim and analyzed using thematic analysis.

Results: There were four main themes for attitude toward the use of AET: 1) benefits of using AET, 2) concerns on taking AET, 3) beliefs on alternative treatment, and 4) beliefs toward the doctor. For practice, six themes were obtained: 1) correct use of AET, 2) appointment adherence, 3) information-seeking behavior, 4) counseling services obtained, 5) experienced side effects of AET, and 6) usage of complementary and alternative medicines.

Conclusion: Several themes concerning attitude and practice of breast cancer patients receiving AET were identified, which may be addressed during treatment consultations in clinical practice.

Keywords: adjuvant endocrine therapy, breast cancer, interview, attitude, practice

\section{Plain Language Summary}

Adjuvant endocrine therapy (AET) is part of the treatment modality for postmenopausal hormone receptor-positive breast cancer patients, which improves overall survival rates. However, its use has been associated with certain side effects that may affect treatment adherence. Therefore, this study was conducted to explore attitude and practice of breast cancer patients while on AET using a semi-structured interview method. Key themes classified under attitudes on using AET include 1) benefits of using AET, 2) concerns on taking AET, 3) beliefs on alternative treatment, and 4) beliefs toward the doctor. Six themes were obtained for practices on using AET, which were 1) correct use of AET, 2) appointment adherence, 3) information-seeking behavior, 4) counseling services obtained, 5) experienced side effects of AET, and 6) usage of complementary and alternative medicines. Understanding patients' attitude and practices on using AET will allow interventions to be designed to optimize treatment adherence for optimal treatment outcome.
Correspondence: Noraida Mohamed

Shah

Centre of Quality Management of Medicines, Faculty of Pharmacy, Universiti Kebangsaan Malaysia, Jalan Raja Muda Abdul Aziz, Kuala Lumpur, 50300 , Malaysia

Tel +60 392898038

Email noraida_mshah@ukm.edu.my 


\section{Introduction}

Breast cancer is the most common cancer affecting women worldwide and is the leading cause of cancer deaths among women in less developed countries. ${ }^{1}$ According to the Malaysian National Cancer Registry Report (2012-2016), the most common cancer in Malaysia was breast cancer, which accounted for $19 \%$ of all cancers. ${ }^{2}$ The use of adjuvant endocrine therapy (AET) has improved the overall survival rates for women with hormone receptorpositive breast cancer. The Malaysian Clinical Practice Guidelines on the Management of Breast Cancer (2010) recommended AET to be given to women with hormone receptor-positive breast cancer. ${ }^{3}$ Recently, the American Society of Clinical Oncology (ASCO) guideline recommended that women with hormone receptor-positive breast cancer receive extended therapy for up to 10 years of AET. $^{4}$

Endocrine therapy prevents the growth of tumor cells by inducing estrogen deprivation. For many years, tamoxifen was the gold standard for AET. In the past decade, third-generation aromatase inhibitors (AIs) began to emerge as an alternative to tamoxifen. Recent clinical trials showed that extended AET with AIs beyond 5 years in postmenopausal women with early breast cancer reduced the occurrence of secondary breast tumors, but the toxicity of adjuvant AIs led to reduced adherence rates, long-term toxicities, and nonbreast cancer-related deaths. Therefore, extended AET is suggested only in women with high-risk early breast cancer who are tolerating treatment. ${ }^{5}$ Trials of AIs in sequential and extended AET following the use of tamoxifen also showed clear benefits of AIs with better side-effect profiles. ${ }^{6,7}$ Due to its mode of action, the use of AET may result in acute and long-term complications secondary to the loss of estrogenic effects such as potentiation of menopausal symptoms. Our previous study found that menopausal symptoms and musculoskeletal pain were common problems encountered by postmenopausal breast cancer patients receiving AET, ${ }^{6}$ but whether these side effects relate to treatment nonadherence was not investigated.

Previous studies suggested that adherence to AET therapy for patients with breast cancer remains suboptimal ${ }^{7-12}$ despite its known efficacy in reducing recurrence and mortality. Multiple independent studies found various factors associated with AET nonadherence. These include the number of comorbidities, ${ }^{11}$ polypharmacy, ${ }^{7}$ age, education level, employment status, presence of side effects, greater concerns about AET, and a lower perceived necessity to take AET. ${ }^{8}$ Acknowledging and addressing these factors is key to providing women with the care needed to improve adherence. ${ }^{9}$ Appropriate interventions can then be instituted to facilitate adherence to AET. Due to the long-term use of AET, patients may have concerns and worries prior to receiving the treatment, which indirectly may affect treatment adherence.

Qualitative studies exploring treatment information needs among women with breast cancer on AET and its use are rather limited. ${ }^{13,14}$ Pieters et al reported that patients on AET desire for more information about potential side effects and symptom management. This has yet to be explored in our local setting. ${ }^{13}$ Therefore, a study exploring on the attitude and practice of breast cancer patients on AET is very much warranted to explore the current issues that affect the attitude and practice among breast cancer patients on AET in Malaysia.

\section{Methods}

\section{Study Design and Setting}

A semi-structured interview method was used to identify the attitude and practice of breast cancer patients toward AET. The study was approved by the Research Ethics Committee of Universiti Kebangsaan Malaysia (Ref No: UKM/PPI/111/8/JEP-2017-596), which was carried out at the Oncology Clinics, Universiti Kebangsaan Malaysia Medical Centre. This study was conducted in accordance with the Declaration of Helsinki.

\section{Participants}

Convenience sampling method was used to select the participants based on the inclusion and exclusion criteria of the study. Postmenopausal breast cancer patients, regardless of the stage of their disease who have taken AET for at least 3 months prior to the interview, were invited to participate in this study during their follow-up appointment with the physicians. The purpose of this study was briefly explained to the patients, and they were given an information sheet regarding this study. Written consent forms were distributed and collected from the participants upon their agreement to take part in the interview. Consent on audio-recording the interview and publication of anonymized responses was obtained from the patients before starting the interview sessions. 


\section{Study Tool}

A semi-structured interview topic guide was used as an outline to explore and inquire more issues concerning the attitude and practice of breast cancer patients on AET. The topic guide interview was developed based on an extensive review of the literature. It revolves around the attitude and practice of breast cancer patients on AET from different aspects, including the duration and the dosage of the drug taken, positive and negative beliefs about the treatment, counseling services, side effects of the treatment, beliefs in complementary and alternative medicines (CAMs), source of the information received, and referral practices. The demographic data of the patients were also collected. The original outline of the topic-guided interview was discussed among the researchers. The finalized topic guide was pre-tested on patients to obtain feedback from patients regarding their opinion on the interview questions.

\section{Interview}

Interviews were conducted from November 2017 to December 2017. The topic guide was utilized to assist the interviewer during the interview with the patients. However, patients have the flexibility to express their opinion regarding the topics brought up by the interviewer. The interviews were conducted until data reached saturation, whereby no new information contributed during the interview. ${ }^{15}$ Each interview was audio-recorded and lasted between 10 and $30 \mathrm{~min}$. The recordings were then transcribed verbatim for analysis.

\section{Data Analysis}

Data were analyzed using the QSR NVivo ${ }^{\circledR} 11$ software program. Constant comparative method with thematic analysis was utilized to analyze all transcribed data. Themes were identified depending on the elements of the dataset. Similar concepts of the parent theme were categorized as subthemes. A four-phase process was used to develop a theme, which includes initialization, construction, rectification, and finalization. ${ }^{16}$ The initialization phase involves reading the whole transcripts of patients to understand the general information and the main issues on the phenomenon under study. This involves creating codes and extracting relevant data to identify important data corresponding to the research question. Sorting out codes and categorizing them into different clusters of codes relevant to the research question constitutes the construction phase.
In this phase, codes with similar concepts to each other were combined for the development of potential themes. Next, the rectification phase involves checking of the potential themes through a discussion among the researchers to ensure all the themes were convincing and answered the research questions. The finalization phase involves a comprehensive analysis of the themes toward understanding the data, by focusing on each theme. This gives a comprehensive view on the study phenomenon. Finalization of an appropriate name for each theme was then carried out and agreed by all researchers. ${ }^{16}$

\section{Results}

\section{Patients' Characteristics}

A total of 30 postmenopausal patients aged between 47 and 84 years with breast cancer on AET for at least 3 months participated in the study. The ethnic distribution consisted of $66.7 \%$ Malays, $30 \%$ Chinese, and 3.3\% Indians. Majority of the patients $(53.3 \%)$ had Stage II breast cancer (Table 1).

\section{Key Themes for Attitude Toward the Use of AET}

There were four main themes for attitude toward the use of AET: 1) benefits of using AET, 2) concerns on taking AET, 3) beliefs on alternative treatment, and 4) beliefs toward the doctor. For practice on using AET, six themes were obtained: 1) correct use of AET, 2) appointment adherence, 3) information-seeking behavior, 4) counseling services obtained, 5) side effects of AET experienced, and 6) usage of CAMs.

\section{Theme I: Benefits of Using AET}

On beliefs toward the medication, few patients mentioned that they strongly believe taking AET and expressed confidence toward AET.

“... will take it (tamoxifen), I believe more on hospital treatment ... When I take tamoxifen, I feel ... what people said secure or more confident ..." (A001, Malay, 47 years, Stage III)

"There is (benefit) ...... it is okay to just take, we don't have any other medication." (A011, Malay, 55 years, Stage III)

Feeling healthy. Some patients said that taking AET made them feel healthier.

"I don't know but I feel a lot better ... I feel a lot better like there is no like urmm lethargic, feel tired no ..." (A022, Malay, 71 years, Stage II) 
Table I Description of the Breast Cancer Patients

\begin{tabular}{|c|c|c|c|c|c|}
\hline Patient & Gender & Age (years) & Ethnicity & Stage of Cancer & Treatment(s) \\
\hline A00I & Female & 47 & Malay & III & Surgery, chemotherapy, radiotherapy, adjuvant endocrine therapy \\
\hline A002 & Female & 63 & Chinese & IV & Surgery, chemotherapy, adjuvant endocrine therapy \\
\hline A003 & Female & 54 & Malay & III & Surgery, chemotherapy, adjuvant endocrine therapy \\
\hline A004 & Female & 64 & Malay & III & Surgery, chemotherapy, radiotherapy, adjuvant endocrine therapy \\
\hline A005 & Female & 71 & Malay & II & Surgery, radiotherapy, adjuvant endocrine therapy \\
\hline A006 & Female & 60 & Malay & II & Surgery, chemotherapy, radiotherapy, adjuvant endocrine therapy \\
\hline A007 & Female & 66 & Malay & II & Surgery, chemotherapy, radiotherapy, adjuvant endocrine therapy \\
\hline A008 & Female & 84 & Chinese & IV & Surgery, radiotherapy, adjuvant endocrine therapy \\
\hline A009 & Female & 53 & Malay & III & Surgery, chemotherapy, radiotherapy, adjuvant endocrine therapy \\
\hline AOIO & Female & 58 & Malay & II & Surgery, chemotherapy, radiotherapy, adjuvant endocrine therapy \\
\hline AOII & Female & 55 & Malay & III & Surgery, adjuvant endocrine therapy \\
\hline $\mathrm{A} 012$ & Female & 52 & Chinese & II & Surgery, chemotherapy, radiotherapy, adjuvant endocrine therapy \\
\hline $\mathrm{A} 013$ & Female & 62 & Chinese & III & Surgery, chemotherapy, radiotherapy, adjuvant endocrine therapy \\
\hline $\mathrm{A} 014$ & Female & 60 & Chinese & II & Surgery, radiotherapy, adjuvant endocrine therapy \\
\hline $\mathrm{A} 015$ & Female & 55 & Malay & II & Surgery, radiotherapy, adjuvant endocrine therapy \\
\hline $\mathrm{A} 016$ & Female & 60 & Malay & IV & Surgery, chemotherapy, radiotherapy, adjuvant endocrine therapy \\
\hline A0I7 & Female & 74 & Malay & I & Surgery, adjuvant endocrine therapy \\
\hline $\mathrm{A} 018$ & Female & 55 & Malay & II & Surgery, chemotherapy, radiotherapy, adjuvant endocrine therapy \\
\hline $\mathrm{A} 019$ & Female & 52 & Malay & II & Surgery, chemotherapy, radiotherapy, adjuvant endocrine therapy \\
\hline A020 & Female & 74 & India & II & Surgery, radiotherapy, adjuvant endocrine therapy \\
\hline A02I & Female & 73 & Chinese & III & Surgery, chemotherapy, adjuvant endocrine therapy \\
\hline A022 & Female & 71 & Malay & II & Surgery, radiotherapy, adjuvant endocrine therapy \\
\hline $\mathrm{A} 023$ & Female & 56 & Malay & IV & Surgery, chemotherapy, radiotherapy, adjuvant endocrine therapy \\
\hline $\mathrm{A} 024$ & Female & 78 & Chinese & II & Surgery, adjuvant endocrine therapy \\
\hline A025 & Female & 74 & Chinese & IV & Surgery, chemotherapy, radiotherapy, adjuvant endocrine therapy \\
\hline A026 & Female & 80 & Chinese & II & Surgery, adjuvant endocrine therapy \\
\hline $\mathrm{A} 027$ & Female & 81 & Malay & II & Surgery, adjuvant endocrine therapy \\
\hline A028 & Female & 56 & Malay & II & Surgery, chemotherapy, adjuvant endocrine therapy \\
\hline A029 & Female & 62 & Malay & II & Surgery, chemotherapy, radiotherapy, adjuvant endocrine therapy \\
\hline $\mathrm{A} 030$ & Female & 48 & Malay & III & Surgery, chemotherapy, radiotherapy, adjuvant endocrine therapy \\
\hline
\end{tabular}

"I feel okay because every time take (AET), my body healthier ...." (A026, Chinese, 80 years, Stage II)

No side effect. Many patients felt that they did not experience any side effects from taking AET and did not affect their general health.

"Yes, it gives me benefits because I didn't get any side effects ..." (A006, Malay, 60 years, Stage II)

" ... there is nothing ... there is no side effect." (A007, Malay, 66 years, Stage II)

"....so far the medicine (AET) ... okay with me." (A014, Chinese, 60 years, Stage II)

“... it (AET) does not give any problem to me." (A017, Malay, 74 years, Stage I)

Lower the chances of breast cancer recurrence. A few patients expressed that the chances of getting breast cancer recurrence is reduced because of taking AET.

"It (AET) lowers this ... the (breast) cancer." (A004, Malay, 64 years, Stage III)
“... maybe there is what umm that does not make me get sick anymore, this thing (breast cancer) not develop anymore ... My body feels lively." (A010, Malay, 58 years, Stage II)

"Alhamdulillah (All praise to God), it looks like health is better." (A016, Malay, 60 years, Stage IV)

\section{Theme 2: Concerns on Taking AET}

Prescription issues. There were patients complaining of the short-treatment duration written on the prescription given to them. As a result, they had to come back to get their medicines every 2-3 months. This poses difficulties to them due to work commitments and the need to travel to the hospital, which is far from their house.

“... not enough (AET) medicine ... it is like this, there is a month with 31 days, right? But they (hospital) only gave 30 pills, so one day is missing a pill ...." (A005, Malay, 71 years, Stage II) 
"... my children always had to take a leave, and it is very difficult because they gave only two boxes (AET)." (A010, Malay, 58 years, Stage II)

Side effects from taking AET. There were patients that experienced at least one side effect from taking the medication, which affected their daily lives. This was contrary to some patients who did not report any side effects from taking AET, as mentioned under Theme 1.

"... then I felt warm in my body and then fatigue ...." (A001, Malay, 47 years, Stage III)

"Taking the medication (AET) somehow made me sweat

a lot ... After that, now if I sweat, there is itchiness ... if,

let us say, I want to lift heavy things, I feel pain (at the joints).” (A005, Malay, 71 years, Stage II)

"I had weight gain ... my clothes were too tight." (A009, Malay, 53 years, Stage III)

"I think I have ... extra discharge ... at night, more itchiness, so I cannot sleep." (A013, Chinese, 62 years, Stage III)

On feeling worried about health in the future, a few patients reported that they felt worried that the breast cancer may recur once the treatment with AET ended.

“ ... feel worried ... there are no medications anymore." (A002, Chinese, 63 years, Stage III)

"I feel worried ... whether it (breast cancer) can occur again or not." (A030, Malay, 48 years, Stage III)

"I feel worried because well, that's the medicine for that kind of disease." (A009, Malay, 53 years, Stage III)

\section{Theme 3: Beliefs on Alternative Treatments}

On beliefs in God or fate, there were patients who felt the importance of trusting God and relying on God's decree. Most of the respondents in this context were Malay Muslims.

"It's called fate, leave it out to Allah (God), we do what the doctor said like (food) abstinence ..." (A016, Malay, 60 years, Stage IV)

"Yes, it is not important to use alternative medicine ... still I believe Allah (God)." (A022, Malay, 71 years, Stage II)

"I do not know because it (breast cancer) is inside, so we do not know what will happen, I hope it (breast cancer) does not happen again, we tawakkal (placing reliance to God).” (A028, Malay, 56 years, Stage II)

On beliefs in CAMs, more than half of the patients used CAMs concurrently with AET. Most of them used ruqyah
(Islamic prayer) as a CAM, and most of them felt that their health becomes better because of it.

“. ... that water (ruqyah) ... Okay ... spiritually of course that thing (ruqyah) helps." (A001, Malay, 47 years, Stage III)

"I feel that with the help from him (Islamic scholar), he also prays, so with the du'a (prayer), I feel that (breast cancer) can heal ... for alternative." (A015, Malay, 55 years, Stage II)

“... I feel no worries because we have other alternatives, we follow the nutrition by Sunnah (ways of the prophet, peace be upon him) practice habbatus sauda (black seed or Nigella sativa), honey, olive oil, or anything that can prevent from (breast cancer) recurrences." (A029, Malay, 62 years, Malay, Stage II)

Despite reports on the benefits of using CAMs, there were patients who reported the worsening of breast cancer due to CAM usage.

"The reason this (breast cancer) became bigger is because I ate belalai gajah (snake grass or Clinacanthus nutans) and spirulina." (A004, Malay, 64 years, Stage III)

\section{Theme 4: Beliefs Toward the Doctor}

Following the doctor's recommendation was the main reason patients take AET. They strongly believed their doctor because they perceived doctors as experts in the field.

"So, if whatever doctor recommend, tamoxifen or any other medication, I will take it ..." (A001, Malay, 81 years, Stage II)

"From my opinion, I follow the doctor's advice. Doctor advised this one (AET)." (A009, Malay, 53 years, Stage III)

"Because we follow what the doctor said, for what exactly." (A015, Malay, 55 years, Stage II)

"... maybe there is (benefit) because the doctor recommends that if we want to heal the cells, we have to take tamoxifen, so I have to ..." (A019, Malay, 52 years, Stage II)

" ... because first it is recommended by the doctor, then this medication must have been researched for us to take ... I feel confident a bit.” (A029, Malay, 62 years, Stage II)

\section{Key Themes for Practices on the Use of AET}

Theme I: Correct Use of AET

Almost all the patients said that they took AET once a day after a meal and had certain mechanisms to ensure that they remember to take the medication. 
“... one (pill) only in the morning. After my breakfast, I take it. ... I think I never forgot (to take medication), and I am always alert." (A020, Indian, 74 years, Stage II)

\section{Theme 2: Appointment Adherence}

All the patients adhered to their appointments with the doctor. The frequency of the appointments ranges from 2 weeks to monthly for new cases and three months to yearly for old cases.

"Six or four months, we always have an appointment date ... yes (always attend the appointment)." (A020, Indian, 74 years, Stage II)

" ... at first it is like weekly, then it becomes two weeks, a month, and then become 3 months once ........always attends it (appointment).” (A021, 73 years, Stage III)

\section{Theme 3: Information-Seeking Behavior}

More than half of the participants reported that they wanted information about AET, particularly on its benefits and side effects.

"I want to know the function of the medicine ... the benefits ... yeah, and the side effects." (A006, Malay, 60 years, Stage II)

“... yes, I want information about it (AET side effects) because I do not know whether this (side effect) comes from tamoxifen or not. I know the (vaginal) discharge is definitely from tamoxifen ... I ask them (the doctor)."

(A027, Malay, 81 years, Stage II)

Sources of information. There were three top sources of information used by the patients, which were through the doctor, the internet, and their family.

"... search through internet and then ask at any support group through WhatsApp regarding their opinion on taking the medication." (A001, Malay, 47 years, Stage III)

"Okay, firstly through doctor, second through Google." (A005, Malay, 71 years, Stage II)

“... pamphlet ... internet ... there is my sister, she gave the information and printed it." (A0015, Chinese, 78 years, Stage II)

\section{Theme 4: Counseling Services Obtained}

There were patients who have undergone counseling services provided by the hospital or the breast cancer association.

"I had attended it (counseling), but I forgot where it happened ... it is about the medication only." (A018, Malay, 55 years, Stage II)

"Yes, I attended it (counseling) when MAKNA (National Cancer Council Malaysia) organized it." (A023, Malay, 56 years, Stage IV)

\section{Theme 5: Experienced Side Effects of AET}

Almost half of the patients informed that they experienced side effects from taking AET. These side effects included hot flashes, joint pain, weight gain, night sweat, dry skin, skin itchiness, and loss of appetite. Minority of the patients felt that the side effects interfered with their daily life. However, they can still manage it.

"There is (side effect) and the body feel hot ... and then fatigue, tired." (A001, Malay, 47 years, Stage III)

"Urmm (There is a side effect) ... I feel hot." (A002, Chinese, 63 years, Stage IV)

"I am not sure if it is because of the medication (AET), right now I feel pain here (bone joint), both of my hands hurt." (A005, Malay, 71 years, Stage II)

"My body gained weight ...." (A009, Malay, 53 years, Stage III)

“... sweat more than normal." (A0021, Chinese, 80 years, Stage II)

\section{Theme 6: Usage of CAMs}

More than half of the participants used CAM while on AET, including ruqyah, herbs, and supplements.

"Yes, I ask Ustaz (Islamic scholar) to recite (Al-Quran, Holy book for Muslims)." (A003, Malay, 54 years, Stage III)

“...... yeah, ... I take Chinese herbs.” (A0021, Chinese, 80 years, Stage II)

“. .. had taken (vitamin) but ... does not know what kind of vitamin it is." (A024, Malay, 71 years, Stage II)

\section{Discussion}

Breast cancer patients commonly received AET as part of their treatment; however, limited studies explore their attitude and practices in using this medication. Understanding their attitude and practices on using AET will enable appropriate interventions to be designed to empower patients in managing their disease. In this study, we identified key themes classified under attitudes and practices on using AET through semi-structured interviews and qualitative analysis methods. Four themes were classified under attitudes on using AET, which were 1) benefits of using AET, 2) concerns on taking AET, 3) beliefs on alternative treatment, and 4) beliefs toward the doctor. Six themes were obtained for practices on using AET, which were 1) correct use of AET, 2) appointment adherence, 3) informationseeking behavior, 4) counseling services obtained, 5) experienced side effects of AET, and 6) usage of CAMs.

Patients in this study affirmed to the benefits of using AET, including lowering the rates of breast cancer 
recurrences, as well as beliefs toward the medications and feeling healthy with no risks of side effects. Nonetheless, some expressed concerns on using AET, including prescription issues, side effects of AET, and feeling worried about their health in the future. Recent ASCO guidelines recommended women with node-positive breast cancers to receive up to a total of 10 years of AET due to its benefit in lowering the risks of breast cancer recurrence and contralateral breast cancer. ${ }^{4} \mathrm{~A}$ benefit in disease-free survival with extended 10 years of AET has also been reported for postmenopausal patients with early breast cancer. ${ }^{17}$ Despite its benefits, patients' beliefs and concerns toward their medications are important aspects to be considered by the healthcare providers. ${ }^{18}$ This should be taken into consideration to enable shared decision-making and ensuring treatment adherence as studies have reported failure to understand patients' perspectives and preferences in treatment decision-making. ${ }^{19}$

A recent local study reported menopausal symptoms and musculoskeletal pain are common side effects of AET experienced by postmenopausal breast cancer patients. ${ }^{6}$ These should be clearly explained to the patients to ensure treatment adherence and ultimately achieve the intended treatment outcome. Prescription issues highlighted by the patients during the interview highlight the need to feed back the information to the prescribers and can easily be rectified through communication between the patients and prescribers. Although this was not a prescribing error, a local study in primary care clinics suggested routine prescriber feedback comprising league tables and a feedback letter to effectively reduce prescribing errors. ${ }^{20}$ Pharmacists may play a role in recommending the appropriate duration to be written on the prescription and dispense enough medicines to ensure treatment adherence.

Patients in this study also had strong faith and reliance to God. Some prayed and utilized CAMs as part of their treatment. Malaysia is a multi-ethnic and multicultural country consisting of Malay, Chinese, Indian, and others with religions that include Islam, Christianity, Buddhism, and Hinduism. ${ }^{21}$ These characteristics are unique for Malaysia unlike many other Western countries. Its religious and cultural diversity may influence beliefs and practices toward the use of CAMs. Studies among breast cancer patients at hospitals in Malaysia identified high prevalence of CAM use and was significantly associated with delay in presentation and resolution of diagnosis. ${ }^{22}$ Farrukh et al identified the most common reason for using CAM among patients with epilepsy was the patients' beliefs on a spiritual or psychological cause of the disease, rather than a neurological disease. ${ }^{23}$
In this study, patients portrayed strong beliefs toward their doctors and followed their advice. Nonetheless, they do indicate a strong desire to know more about their AET and tend to seek information from their doctor, the internet, and their family. A recent local study using the European Organisation for the Research and Treatment of Cancer Quality of Life Questionnaire Information Module reported that cancer patients had moderate levels of information and high satisfaction on the information received. However, most wanted more information. In the same study, doctors, nurses, and family members were the most useful sources of information. ${ }^{24}$ Knowledge and adherence to AET improves when patients were given information related to AET's mode of action and potential side effects. ${ }^{25}$ It is imperative that breast cancer patients are empowered with information pertaining to their disease and its treatment to ensure successful therapy. This can be done through shared decisionmaking, a process by which medical decisions with multiple appropriate options are made together by clinicians and patients, considering patients' values and preferences. ${ }^{26,27}$ This can be facilitated using patient decision aids, which can assist patients in exploring clinical evidence, clarify and deliberate their preferences for options, and prepare them to communicate with their healthcare practitioners. ${ }^{28}$

There are few limitations in this study. It was conducted at a single tertiary care hospital setting at an urban area and therefore may not represent all breast cancer patients on AET in Malaysia. Furthermore, convenience sampling was used to select participants for this interview. Therefore, only patients who are willing to be interviewed were included and may have resulted in selection bias. Nonetheless, this study provided useful insights into the attitudes and practices of postmenopausal breast cancer patients on AET.

\section{Conclusion}

Postmenopausal breast cancer patients had strong beliefs on the benefits of AET. They had some concerns with its side effects and worries that the cancer may recur once treatment is completed. They had contrasting beliefs on CAMs based on their own experiences and religious practices. Patients expressed the need for further information pertaining to their treatment. Thus, the development of an information support tool is important to empower breast cancer patients receiving AET. Findings from this study may also be utilized in the development of a survey instrument to determine attitudes and practices on the use of AET among a larger number of breast cancer patients. 


\section{Acknowledgments}

We would like to thank all the participants, nurses and administrative personnel at UKM Medical Centre for assisting in this study.

\section{Disclosure}

The authors have no financial or other conflicts of interest to disclose. The study received financial support from Universiti Kebangsaan Malaysia (GUP-2020-004).

\section{References}

1. Bray F, Ferlay J, Soerjomataram I, et al. Global cancer statistics 2018: GLOBOCAN estimates of incidence and mortality worldwide for 36 cancers in 185 countries. CA Cancer J Clin. 2018;68 (6):394-424. doi:10.3322/caac. 21492

2. Azizah AM, Hashimah B, Nirmal K, et al. Malaysian National Cancer Report (MNCR) 2012-2016. Malaysia: Ministry of Health; 2019. Available from: https://drive.google.com/file/d/ 1BuPWrb05N2Jez6sEP8VM5r6JtJtlPN5W/view. Accessed January 21, 2021.

3. Malaysia clinical practice guidelines: management of breast cancer. Malaysia: Ministry of Health; 2010. Available from: https://www. moh.gov.my/moh/attachments/6915.pdf. Accessed January 21, 2021.

4. Burstein HJ, Lacchetti C, Anderson $\mathrm{H}$, et al. Adjuvant endocrine therapy for women with hormone receptor-positive breast cancer: ASCO clinical practice guideline focused update. J Clin Oncol. 2019;37(5):423-438. doi:10.1200/JCO.18.01160

5. van Hellemond IEG, Geurts SME, Tjan-Heijnen VCG. Current status of extended adjuvant endocrine therapy in early stage breast cancer. Curr Treat Options Oncol. 2018;19(5):26. doi:10.1007/s11864-0180541-1

6. Choo SB, Saifulbahri A, Zullkifli SN, et al. Adjuvant endocrine therapy side-effects among postmenopausal breast cancer patients in Malaysia. Climacteric. 2019;22(2):175-181. doi:10.1080/13697137.2018.1540563

7. Calip GS, Xing S, Jun D-H, et al. Polypharmacy and adherence to adjuvant endocrine therapy for breast cancer. J Oncol Pract. 2017;13 (5):e451-e462. doi:10.1200/JOP.2016.018317

8. Brett J, Fenlon D, Boulton M, et al. Factors associated with intentional and unintentional non-adherence to adjuvant endocrine therapy following breast cancer. Eur J Cancer Care (Engl). 2018;27(1): e12601. doi:10.1111/ecc.12601.

9. Lambert LK, Balneaves LG, Howard AF, et al. Patient-reported factors associated with adherence to adjuvant endocrine therapy after breast cancer: an integrative review. Breast Cancer Res Treat. 2018;167(3):615-633. doi:10.1007/s10549-017-4561-5

10. Farias AJ, Du XL. Association between out-of-pocket costs, race/ ethnicity, and adjuvant endocrine therapy adherence among medicare patients with breast cancer. J Clin Oncol. 2017;35(1):86-95. doi:10.1200/JCO.2016.68.2807

11. Ali EE, Cheung KL, Lee CP, et al. Prevalence and determinants of adherence to oral adjuvant endocrine therapy among breast cancer patients in Singapore. Asia Pac J Oncol Nurs. 2017;4(4):283-289. doi:10.4103/2347-5625.212864

12. Quinn EM, Fleming C, O'Sullivan MJ. Endocrine therapy adherence: a cross-sectional study of factors affecting adherence and discontinuation of therapy. Ir J Med Sci. 2016;185(2):383-392. doi:10.1007/ s11845-015-1307-4
13. Pieters HC, Green E, Khakshooy S, et al. A qualitative comparison of how older breast cancer survivors process treatment information regarding endocrine therapy. PLoS One. 2019;14(1):e0210972. doi:10.1371/journal.pone. 0210972

14. Moon Z, Moss-Morris R, Hunter MS, et al. Understanding tamoxifen adherence in women with breast cancer: a qualitative study. $\mathrm{Br}$ J Health Psychol. 2017;22(4):978-997. doi:10.1111/bjhp.12266

15. Guest G, Bunce A, Johnson L. How many interviews are enough? An experiment with data saturation and variability. Field Methods. 2006;18(1):59-82. doi:10.1177/1525822X05279903

16. Vaismoradi M, Jones J, Turunen H, et al. Theme development in qualitative content analysis and thematic analysis. $J$ Nurs Educ Pract. 2016;6(5):100-110.

17. Li L, Chang B, Jiang X, et al. Clinical outcomes comparison of 10 years versus 5 years of adjuvant endocrine therapy in patients with early breast cancer. BMC Cancer. 2018;18(1):977. doi:10.1186/s12885-018-4878-4

18. Horne R, Chapman SC, Parham R, et al. Understanding patients' adherence-related beliefs about medicines prescribed for long-term conditions: a meta-analytic review of the necessity-concerns framework. PLoS One. 2013;8(12):e80633. doi:10.1371/journal.pone.0080633

19. Nies YH, Islahudin F, Chong WW, et al. Treatment decision-making among breast cancer patients in Malaysia. Patient Prefer Adherence. 2017;11:1767-1777. doi:10.2147/PPA.S143611

20. Lim WY, Hss AS, Ng LM, et al. The impact of a prescription review and prescriber feedback system on prescribing practices in primary care clinics: a cluster randomised trial. BMC Fam Pract. 2018;19 (1):120. doi:10.1186/s12875-018-0808-4

21. Department of statistic Malaysia, current population estimates, Malaysia, 2018-2019. Available from: https://www.dosm.gov.my/v1/index.php?r= c o 1 u m n / c the me B y Cat \& c a t $=155 \&$ bu $1_{-}$i d $=$ aWJZRkJ4UEdKcUZpT2tVT090Snpydz09\&menu_id= L0pheU43NWJwRWVSZk1WdzQ4TlhUUT09\#: :text=Malaysia's\% 20population\%20in\%202019\%20is, 1.1\%20per\%20cent\%20in\%202018. Accessed January 21, 2021.

22. Mohd Mujar NM, Dahlui M, Emran NA, et al. Complementary and alternative medicine (CAM) use and delays in presentation and diagnosis of breast cancer patients in public hospitals in Malaysia. PLoS One. 2017;12(4):e0176394. doi:10.1371/journal.pone.0176394

23. Farrukh MJ, Makmor-Bakry M, Hatah E, et al. Use of complementary and alternative medicine and adherence to antiepileptic drug therapy among epilepsy patients: a systematic review. Patient Prefer Adherence. 2018;12:2111-2121. doi:10.2147/PPA.S179031

24. Lew YL, Ismail F, Abdul Aziz SA, et al. Information received and usefulness of the sources of information to cancer patients at a tertiary care centre in Malaysia. J Cancer Educ. 2019. doi:10.1007/s13187-019-01637-9

25. Heisig SR, Shedden-Mora MC, von Blanckenburg P, et al. Informing women with breast cancer about endocrine therapy: effects on knowledge and adherence. Psychooncology. 2015;24(2):130-137. doi:10.1002/pon.3611

26. Härter M, Moumjid N, Cornuz J, et al. Shared decision making in 2017: international accomplishments in policy, research and implementation. Z Evid Fortbild Qual Gesundhwes. 2017;123:1-5. doi:10.1016/j.zefq.2017.05.024

27. Latenstein CSS, Thunnissen FM, Thomeer BJM, et al. The association between patients' preferred treatment after the use of a patient decision aid and their choice of eventual treatment. Health Expect. 2020;23 (3):651-658.

28. Stacey D, Legare F, Col NF, et al. Decision aids for people facing health treatment or screening decisions. Cochrane Database Syst Rev. 2014;1:CD001431. 


\section{Publish your work in this journal}

Patient Preference and Adherence is an international, peer-reviewed, open access journal that focusing on the growing importance of patient preference and adherence throughout the therapeutic continuum. Patient satisfaction, acceptability, quality of life, compliance, persistence and their role in developing new therapeutic modalities and compounds to optimize clinical outcomes for existing disease

states are major areas of interest for the journal. This journal has been accepted for indexing on PubMed Central. The manuscript management system is completely online and includes a very quick and fair peer-review system, which is all easy to use. Visit http:// www.dovepress.com/testimonials.php to read real quotes from published authors. 